\title{
Cancer genomics: opportunities for medicinal chemistry?
}

\begin{abstract}
"Although the advances in cancer genomics research have produced an extremely large volume of information on potential therapeutic pathways and targets, there is still a gap in the translation of knowledge from bench to bedside."
\end{abstract}

First draft submitted: 1 January 2016; Accepted for publication: 12 January 2016; Published online: 15 March 2016

Keywords: big data $\bullet$ cancer genomics $\bullet$ driver mutation $\bullet$ drug combination $\bullet$ drug target

\section{Cancer genomics}

It is well known that cancer is a genetic disease. Cancer genomics is to systematically sequence the genome in order to identify recurrent genomics alterations in cancers [1]. To date, more than 80 types of cancers are, or have been, sequenced by centers around the world. The Cancer Genome Atlas (TCGA) [2] project and the International Cancer Genome Consortium (ICGC) [3] project are among the most renowned. These projects have expanded the list of cancer genes, and also revealed many emerging disregulated cellular processes such as those involved in chromatin and epigenomic regulation, as well as those that are involved in RNA splicing, protein homeostasis, metabolism and lineage maturation. For a more detailed review on cancer genomics, readers are referred to the review articles $[1,4]$.

The rapid advance in cancer genomics research can be largely attributed to the application of novel next-generation sequencing (NGS) technologies and the development of new computational tools.

\section{NGS for cancer genomics research}

NGS has revolutionized cancer genomics research. Through whole-genome sequencing, cancer genomic alterations, including point mutations, small insertions or deletions, copy number alternations and structural variations can be identified. While the whole-transcriptome sequencing approaches can quantify gene expression profiles and alternative splicing, as well as RNA editing and fusion transcripts, Bisulfite-Seq and ChIP-seq technologies can be utilized to investigate the epigenetic alterations, such as DNA methylation change, histone modifications and transcription factor binding sites. For example, in the pilot project of TCGA, the analysis of DNA copy number, gene expression, DNA methylation aberrations and nucleotide sequence aberrations were done on 206 glioblastomas with the first generation of sequencing technologies [5]. In the recent TCGA publication [6], comprehensive molecular analysis of 333 primary prostate carcinomas were performed on multiple NGS platforms and other assays for detecting mutations, copy number alterations, mRNA expression fusions, DNA methylation and miRNA expression as well as protein expression.

\section{Computational tools for cancer genomics research}

Computational tools enable the powerful analysis of large volume of cancer genomics data to better understand the mechanisms of cancer. 'Driver' mutations are those alterations in genes that enable cancer cells gain advantage against their surrounding cells. These mutations can significantly influence the key pathways regulating cell cycles, survival and genome stability. During cancer progress, a lot of 'passenger' mutations can

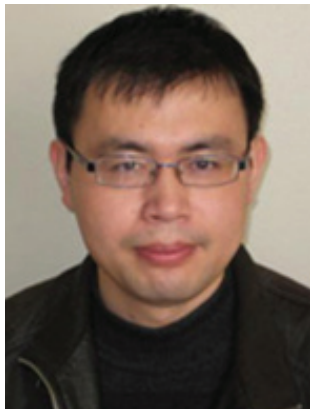

Lirong Wang Department of Pharmaceutical Sciences, School of Pharmacy, Computational Chemical Genomics Screening Center, NIH National Center of Excellence for Drug Abuse Research, University of Pittsburgh, PA 15261, USA

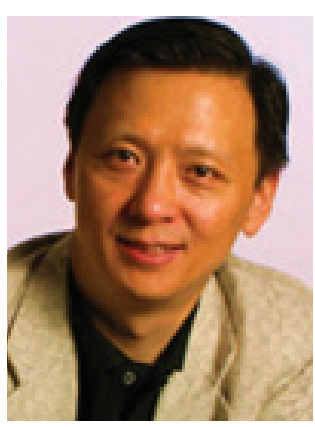

Xiang-Qun Xie

Author for correspondence: Department of Pharmaceutical Sciences, School of Pharmacy, Computational Chemical Genomics Screening Center, NIH National Center of Excellence for Drug Abuse Research, Drug Discovery Institute, University of Pittsburgh, Pittsburgh, PA 15261, USA

Tel.: +1 4123835276

xix15@pitt.edu

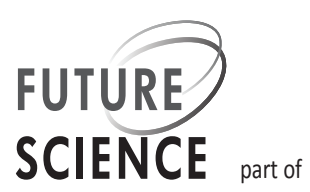


co-occur with driver mutation due to genome instability or quick proliferation and DNA repair deficiency of cancer cells. Though the Pan-Cancer analysis [7], scientists now can categorize those driver mutations and further delineate their roles in cancer initiation, proliferation and migration. For example, by the combination of OncodriveFM and OncodriveCLUST in the IntOGen-mutations pipeline [8], Tamborero et al. identified a list of 291 high-confidence cancer driver genes from 3205 tumors of 12 different cancer types [9].

\section{Cancer genomics data sharing}

After comprehensive genomic characterization and analysis of each cancer, the comprehensive data that have been generated by cancer genomics projects are freely available through public data portals such as cBioPortal [10] and Firehose [11]. The cBioPortal provides visualization, analysis and download of large-scale cancer genomics data sets from 105 cancer genomics studies. The query interface enables researchers to interactively explore the relationship between genetic alterations and clinical outcomes. The Firehose project contributed by Broad Institutes provides a platform of computational pipelines for cancer genomics analysis and it makes TCGA preprocessed data publicly available via web services and data portals.

For scientists working on field of medicinal chemistry, the cancer genomics data provides challenges and opportunities for designing new anticancer drugs.

\section{"The heterogeneity of cancer cells contributes to drug resistance."}

\section{New drug targets}

As we mentioned above, these cancer genomics projects generate potential drug targets. As estimated by Carlota Rubio-Perez et al., only 5.9\% cancers are tractable by approved agents and up to $73.3 \%$ could benefit from agents in clinical stages [12]. The latter statement is promising, however, as the failure rate is high for clinical trials of anticancer drugs, there are still unmet needs for novel drugs modulating the newly discovered targets. To determine which potential targets should be prioritized for therapy, it is suggested that scientists integrate multiple lines of evidence, such as data from RNAi and small-molecule screens to confirm the roles of the new targets on the subtype of cancer that is of interest. The Sanger project, Genomics of Drug Sensitivity in Cancer, has tested 140 anticancer drugs across almost 700 cancer cell lines, and the correlated drug sensitivity data with genomic datasets have been analyzed to identify molecular features associated with drug sensitivity and resistance [13]. In addition, the Achilles Project of Broad Institute has screened shRNA on 11,000 genes in 216 cancer cell lines to identify genes that are required for cell proliferation and/or viability [14]. Such data will provide valuable clues to validate targets as these cell lines carry diverse mutations. Using the shRNA-based loss-of-function data, Cannon et al. recently identified an 'exceptional responder' cell line to MEK1 Inhibition [15].

\section{New drug combination}

The heterogeneity of cancer cells contributes to drug resistance. Also most cancers have two to six of significantly mutated genes [16]. Rational drug combinations to inhibit multiple survival pathways in cancer cells can counter drug resistance. Designing drugs with polypharmacophore features that can simultaneously target multiple pathways for cancer treatment is still a challenge. AstraZeneca has released some drug combinations data, including approximately $11.5 \mathrm{k}$ experimental tests of over 118 drugs on 85 cancer cell lines, and monotherapy drug response data for each drug and cell line [17]. Such data, together with the cancer genomics data, will benefit the rational design of multiple targeted drugs. It is worth mentioning that, although recent cancer immunotherapy has greatly advanced in the treatment of some cancers, the potential of therapeutic resistance still exists. Chemical compounds that can work in synergy with immunotherapy have the potential to enhance the anticancer immune response.

\section{New cancer subtypes \& models}

The cancer genomics data has also revealed new cancer subtypes and provided new cancer models for anticancer drug research. Human cancer cell lines have been widely used in cancer biology and drug discovery. The Cancer Cell Line Encyclopedia (CCLE) project has genetically characterized more than 1000 human cancer cell lines for their DNA copy number, mRNA expression and mutations [18]. With these data, medicinal chemists can identify suitable models of cell lines to test their drugs for targeted cancer therapies. These cell lines exhibit desirable genetic features that more closely resemble cognate cancer profiles and can even be from multiple cancer types. Since some pronounced differences in molecular profiles between commonly used cancer cell lines and cancer samples from patients have been reported [19], patient-derived cell lines and patient-derived xenografts [20], as an in vitro and an in vivo models, respectively, have been deemed more reliable to predict mechanisms of drug resistance and inform therapeutic strategy design. 


\section{Conclusion}

Although the advances in cancer genomics research have produced an extremely large volume of information on potential therapeutic pathways and targets, there is still a gap in the translation of knowledge from bench to bedside. Medicinal chemists are encouraged to harness these valuable tools and resources to rationally design novel compounds for those newly identified targets or to optimize their leads by precisely targeting the cancer types with distinct genetic features.

\section{References}

1 Garraway LA, Lander ES. Lessons from the cancer genome. Cell 153(1), 17-37 (2013).

2 The Cancer Genome Atlas (TCGA). http://cancergenome.nih.gov

3 The International Cancer Genome Consortium (ICGC). https://icgc.org

4 Vogelstein B, Papadopoulos N, Velculescu VE, Zhou S, Diaz LA, Kinzler KW. Cancer genome landscapes. Science 339(6127), 1546-1558 (2013).

5 Mclendon R, Friedman A, Bigner D et al. Comprehensive genomic characterization defines human glioblastoma genes and core pathways. Nature 455(7216), 1061-1068 (2008).

6 Network CGaR. The molecular taxonomy of primary prostate cancer. Cell 163(4), 1011-1025 (2015).

7 Weinstein JN, Collisson EA, Mills GB et al. The cancer genome atlas pan-cancer analysis project. Nat. Genet. 45(10), 1113-1120 (2013).

8 Gonzalez-Perez A, Perez-Llamas C, Deu-Pons J et al. IntOGen-mutations identifies cancer drivers across tumor types. Nat. Methods 10(11), 1081-1082 (2013).

9 Tamborero D, Gonzalez-Perez A, Perez-Llamas C et al. Comprehensive identification of mutational cancer driver genes across 12 tumor types. Sci. Rep. 3, 2650 (2013).

10 Gao J, Aksoy BA, Dogrusoz U et al. Integrative analysis of complex cancer genomics and clinical profiles using the cBioPortal. Sci. Signal. 6(269), pl1-pl1 (2013).

11 Marx V. Drilling into big cancer-genome data. Nat. Methods 10(4), 293-297 (2013).

\section{Financial \& competing interest disclosure}

The authors would like to acknowledge the financial support for the laboratory at University of Pittsburgh from the NIH grants R21HL109654 and P30DA035778 (X-Q Xie). The authors have no other relevant affiliations or financial involvement with any organization or entity with a financial interest in or financial conflict with the subject matter or materials discussed in the manuscript apart from those disclosed.

No writing assistance was utilized in the production of this manuscript.

12 Rubio-Perez C, Tamborero D, Schroeder MP et al. In silico prescription of anticancer drugs to cohorts of 28 tumor types reveals targeting opportunities. Cancer Cell 27(3), 382-396 (2015).

13 Garnett MJ, Edelman EJ, Heidorn SJ et al. Systematic identification of genomic markers of drug sensitivity in cancer cells. Nature 483(7391), 570-575 (2012).

14 Cowley GS, Weir BA, Vazquez F et al. Parallel genomescale loss of function screens in 216 cancer cell lines for the identification of context-specific genetic dependencies. Sci. Data 1, 140035 (2014).

15 Gannon HS, Kaplan N, Tsherniak A et al. Identification of an "exceptional responder" cell line to MEK1 inhibition: clinical implications for MEK-targeted therapy. Mol. Cancer Res. 14(2), 207-215 (2015) (Epub ahead of print).

16 Kandoth C, Mclellan MD, Vandin F et al. Mutational landscape and significance across 12 major cancer types. Nature 502(7471), 333-339 (2013).

17 AstraZeneca-Sanger Drug Combination Prediction DREAM Challenge. www.synapse.org/\#!Synapse:syn4231880

18 Barretina J, Caponigro G, Stransky N et al. The Cancer Cell Line Encyclopedia enables predictive modelling of anticancer drug sensitivity. Nature 483(7391), 603-607 (2012).

19 Domcke S, Sinha R, Levine DA, Sander C, Schultz N. Evaluating cell lines as tumour models by comparison of genomic profiles. Nat. Comm. 4 (2013).

20 Tentler JJ, Tan AC, Weekes CD et al. Patient-derived tumour xenografts as models for oncology drug development. Nat. Rev. Clin. Oncol. 9(6), 338-350 (2012). 